\title{
Nuclear forward scattering application to the spiral magnetic structure study in $\epsilon-\mathrm{Fe}_{2} \mathrm{O}_{3}$
}

\author{
Yu. V. Knyazev $\odot,{ }^{1, *}$ A. I. Chumakov $\odot,{ }^{2, \dagger}$ A. A. Dubrovskiy, ${ }^{1}$ S. V. Semenov, ${ }^{1}$ I. Sergueev, ${ }^{3}$ S. S. Yakushkin, ${ }^{4}$ \\ V. L. Kirillov, ${ }^{4}$ O. N. Martyanov, ${ }^{4}$ and D. A. Balaev ${ }^{1}$ \\ ${ }^{1}$ Kirensky Institute of Physics, Federal Research Center KSC SB RAS, Akademgorodok 50, Building 38, Krasnoyarsk, 660036 Russia \\ ${ }^{2}$ ESRF-The European Synchrotron, 71 Avenue des Martyrs CS40220, F-38043 Grenoble Cedex 9, France \\ ${ }^{3}$ Deutsches Elektronen-Synchrotron DESY, 22607, Hamburg, Germany \\ ${ }^{4}$ Boreskov Institute of Catalysis, Russian Academy of Sciences, Siberian Branch, Novosibirsk, 630090 Russia
}

(Received 28 November 2019; accepted 19 February 2020; published 6 March 2020)

\begin{abstract}
The $\epsilon-\mathrm{Fe}_{2} \mathrm{O}_{3}$ magnetic structure has been analyzed using the synchrotron radiation source. Time spectra of nuclear forward scattering for isolated nanoparticles with an average size of $8 \mathrm{~nm}$ immobilized in a xerogel matrix have been recorded in the temperature range of $4-300 \mathrm{~K}$ in applied magnetic fields of $0-4 \mathrm{~T}$ in the longitudinal direction at the European Synchrotron Radiation Facility (ESRF, Grenoble, France). It has been found that the external magnetic field does not qualitatively change the $H_{\mathrm{hf}}(\mathrm{T})$ behavior, but makes a strong opposite impact on the hyperfine fields in the nonequivalent iron sites, leading to the divergence of $H_{\mathrm{hf}}$ polar angle dependences below $80 \mathrm{~K}$. A complete diagram of the $\epsilon-\mathrm{Fe}_{2} \mathrm{O}_{3}$ magnetic structure in the temperature range of $4-300 \mathrm{~K}$ is proposed. At $300 \mathrm{~K}$, the $\epsilon-\mathrm{Fe}_{2} \mathrm{O}_{3}$ compound is confirmed to be a collinear ferrimagnet. The experimental results show that the magnetic transition at $150-80 \mathrm{~K}$ leads to the formation of a noncollinear magnetic structure. Furthermore, in the range of the 80-4 K, the ground state of a magnetic spiral is established. The experimental results are supplemented by the analysis of the exchange interactions and temperature dependence of the magnetization in a magnetic field of $7 \mathrm{~T}$.
\end{abstract}

DOI: 10.1103/PhysRevB.101.094408

\section{INTRODUCTION}

The $\epsilon-\mathrm{Fe}_{2} \mathrm{O}_{3}$ oxide nanoparticles are a unique material with intriguing magnetic properties that has been the focus of research for the past two decades. Despite using different modern techniques [1-7], the type of a magnetic structure of this material is still unclear. The $\epsilon-\mathrm{Fe}_{2} \mathrm{O}_{3}$ magnetic structure undergoes a complex evolution, which especially concerns the well-known transition at $80-150 \mathrm{~K}$ [7]. This transition is accompanied by a huge change in the coercivity $[7,8]$. In view of recent developments [9], the temperature of the transition to the paramagnetic state seems ambiguous. According to the early data $[10,11]$, this temperature is $500 \mathrm{~K}$, but a thorough examination of the magnetic properties shows that it can be about $800 \mathrm{~K}$, which was noticed first in Ref. [12] during the magnetization and electron spin resonance measurements and then observed in later works $[9,13]$.

The magnetic structure of $\epsilon-\mathrm{Fe}_{2} \mathrm{O}_{3}$ around the magnetic transition at $80-150 \mathrm{~K}$ is difficult to determine because $\epsilon-\mathrm{Fe}_{2} \mathrm{O}_{3}$ is a nanoscale material, which can only be obtained in the form of nanoparticles [14,15], nanotubes [16], or thin films [17]. The nanocrystallinity of this material leads to a strong broadening of diffraction peaks and, consequently, to their overlap, which decreases a number of effectively available neutron diffraction reflections [7]. Furthermore, a complex crystal structure with different local surroundings of

\footnotetext{
*yuk@iph.krasn.ru

†chumakov@esrf.fr
}

iron atoms complicates the interpretation of the experimental data. The magnetic structure is unanimously considered to be collinear and ferrimagnetic above $150 \mathrm{~K}$ (although canting of the magnetic moments of the third and fourth sublattices is determined), whereas at lower temperatures the structure is interpreted using two different models.

The first model suggests that the magnetic structure represents a complex spiral structure incommensurate with the crystal lattice $[7,18]$. Although the neutron diffraction data were thoroughly analyzed, the attempts to unambiguously take into account all the factors in the spiral magnetic structure model were unsuccessful. It was mentioned that the spiral magnetic structures that could be compatible with the observed narrow Mössbauer lines of the low-temperature phase are inconsistent with the neutron diffraction data. Therefore, in order to match the results of different techniques, a rectangular modulated structure was proposed, which represents a superposition of a series of sinusoidally modulated structures with harmonics as propagation vectors.

The alternative model is based primarily on the Mössbauer spectroscopy data obtained in an applied magnetic field and the magnetization measurements. According to these data, the magnetic structure can be described as a canted antiferromagnet $[3,4]$ and the magnetic transition in the temperature range of $80-150 \mathrm{~K}$ results in the change of the canting angle between the magnetic moments. The rotation of the magnetic moment in the tetrahedral sites during the magnetic transition was confirmed also by conventional Mössbauer spectroscopy and nuclear forward scattering of synchrotron radiation in zero magnetic field [19]. 
At present, it is known for certain that the $\epsilon-\mathrm{Fe}_{2} \mathrm{O}_{3}$ oxide crystal structure contains four nonequivalent iron sites (octahedral distorted Fe-D1 and Fe-D2, octahedral undistorted $\mathrm{Fe}-\mathrm{R}$, and tetrahedral FeT) [1,2]. In the Mössbauer study, the designation of the nonequivalent sites is somewhat different and hereinafter given in accordance with Refs. [3,4]. Specifically, the distorted octahedral Fe-D1 and Fe-D2 sites are hereinafter referred to as $\mathrm{Fe} 2$ and $\mathrm{Fe} 3$, respectively, and the $\mathrm{Fe}-\mathrm{R}$ and $\mathrm{Fe}-\mathrm{T}$ sites as $\mathrm{Fe} 1$ and $\mathrm{Fe} 4$, respectively. As was shown in Refs. [3-5,7], the tetrahedral iron site Fe4 strongly affects the magnetic behavior of the nanocrystalline $\epsilon-\mathrm{Fe}_{2} \mathrm{O}_{3}$ oxide.

In Ref. [18], the authors studied the interplay of the magnetic transition and local surrounding of iron atoms in the nonequivalent crystallographic sites. It was established that the $\epsilon-\mathrm{Fe}_{2} \mathrm{O}_{3}$ oxide undergoes a second-order structural transition caused by the simultaneous coordination change in one of the octahedral Fe-D1 sites and in the tetrahedral FeT site. This leads to the change in the charge density due to a significant (by about 10\%) decrease in the $\mathrm{Fe}-\mathrm{O}$ bond length. The x-ray magnetic circular dichroism (XMCD) data show that this transition changes the spin-orbit coupling because of the instability of the orbit contribution to the iron magnetic moment. The spin component of the iron magnetic moment remains temperature independent in the temperature region of the magnetic transition, and the orbital component of the magnetic moment first decreases, attains its minimum value at $\approx 120 \mathrm{~K}$, and then increases to the values observed above the transition at $200 \mathrm{~K}$.

Certainly, the study of the $\epsilon-\mathrm{Fe}_{2} \mathrm{O}_{3}$ magnetic structure are not limited by the experimental investigations. In particular, in one of the first attempts to theoretically describe the magnetic structure, the values of the indirect exchange interactions were determined in the framework of the molecular field model without taking into account the intrasublattice exchange $[20,21]$. The results are qualitatively consistent with the experimental data reported in Refs. [7,18]. The $\epsilon-\mathrm{Fe}_{2} \mathrm{O}_{3}$ magnetic structure determined by the theoretical calculations is ferrimagnetic due to the incomplete compensation of the iron magnetic moments in sites 1 and 4 (Fe-R and Fe-T). The iron magnetic moments in sites 2 and 3 (Fe-D1and Fe-D2) are antiparallel and equal to each other.

Therefore, the determination of the complex behavior of the $\epsilon-\mathrm{Fe}_{2} \mathrm{O}_{3}$ compound becomes a nontrivial task. The nanoscale origin demands precision experimental techniques highl sensitive to any changes in the magnetic structure to be used. The synchrotron radiation source is the most suitable tool for studying nanoobjects. In this work, we used the synchrotron radiation source to clarify the effect of the structural transition at $80-150 \mathrm{~K}$ on the magnetic structure and studied the temperature dependence of magnetization and nuclear forward scattering (NFS). The investigations were carried out on synthesized ultrafine $\epsilon-\mathrm{Fe}_{2} \mathrm{O}_{3}$ nanoparticles using linearly polarized synchrotron radiation in the temperature range of $4-300 \mathrm{~K}$ in applied magnetic fields of $0-4 \mathrm{~T}$. The NFS technique with the nuclear scattering measurement on the timescale was chosen because it allows one to distinguish the ferro- and antiferromagnetic structures of the samples [22], which is impossible in conventional Mössbauer spectroscopy in the energy scale.

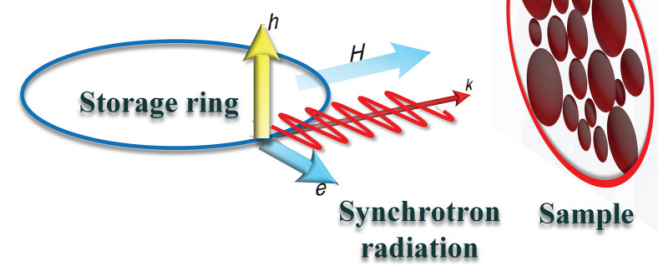

FIG. 1. Geometry of the experiment. The blue circumference schematically shows the storage ring. The arrows denoted by $\mathbf{h}, \mathbf{e}, \mathbf{H}$, and $\mathbf{k}$ show the electric, magnetic, and wave vector of synchrotron radiation and the external magnetic field, respectively. The sample plane is perpendicular to the synchrotron radiation.

\section{EXPERIMENTAL METHODS}

We investigated $\epsilon-\mathrm{Fe}_{2} \mathrm{O}_{3}$ nanoparticles (20 wt.\%) immobilized in a xerogel matrix. The synthesis was performed by embedding the $\mathrm{Fe}^{2+}$ salt $\mathrm{FeSO}_{4} \times 7 \mathrm{H}_{2} \mathrm{O}$ into the $\mathrm{SiO}_{2}$ hydrogel by diffusion exchange in the $\mathrm{H}_{2} \mathrm{SO}_{4}(\mathrm{pH}=2)$ aqueous solution, followed by annealing at $900{ }^{\circ} \mathrm{C}$ in air. The details of the synthesis were described in Ref. [23]. The high resolution transmission electron microscopy (HRTEM) and $\mathrm{x}$-ray diffraction (XRD) investigations of the sample proved the formation of $\epsilon-\mathrm{Fe}_{2} \mathrm{O}_{3}$ nanoparticles in the silica matrix. Neither noticeable admixtures of other iron oxide polymorphs nor agglomeration of the nanoparticles upon annealing were found. According to the HRTEM data, the average nanoparticle size was $8 \mathrm{~nm}$ with a standard deviation of $3.4 \mathrm{~nm}$ [23].

For the synchrotron radiation experiment, the xerogel with the embedded $\epsilon-\mathrm{Fe}_{2} \mathrm{O}_{3}$ nanoparticles was ground and the obtained powder was poured into cylindrical holes with a diameter of $3 \mathrm{~mm}$ in a 2-mm-thick aluminum plate. The NFS study was carried out at the nuclear resonance beamline [24] ID18 of the European Synchrotron Radiation Facility. The synchrotron radiation was linearly $\sigma$ polarized. The measurements were performed in the temperature range of 4-300 K in external magnetic fields of $0-4 \mathrm{~T}$ using a cryo-magnetic system. The external field was applied along the x-ray beam. This experimental geometry allows one to distinguish the ferro- and antiferromagnetic structures [22]. The experimental scheme is sketched out in Fig. 1.

The ESRF storage ring operated in a four-bunch mode, emitting short $(\approx 0.1 \mathrm{~ns})$ synchrotron radiation pulses with a period of $704 \mathrm{~ns}$. Such a time window is convenient for measuring the time spectra of nuclear scattering for the ${ }^{57} \mathrm{Fe}$ Mössbauer isotope with a natural lifetime of $141 \mathrm{~ns}$. The iron nuclei in the samples were excited by a beam of linearly polarized $\mathrm{x}$ rays with an energy of $14.4125 \mathrm{keV}$ of the first excited state of the ${ }^{57} \mathrm{Fe}$ nucleus. The $\mathrm{x}$-ray beam was monochromatized to an energy bandwidth of $\approx 0.7 \mathrm{meV}$, which is much greater than the characteristic hyperfine splitting of the nuclear levels $(<1 \mu \mathrm{eV})$.

The time distributions (time spectra) of time-delayed reemitted $\gamma$ rays were measured using fast avalanche photodiode detectors [25] with a time resolution of $\approx 1 \mathrm{~ns}$. The zero-time reference was marked by a moment of detecting the prompt electron scattering. The measured time spectra 

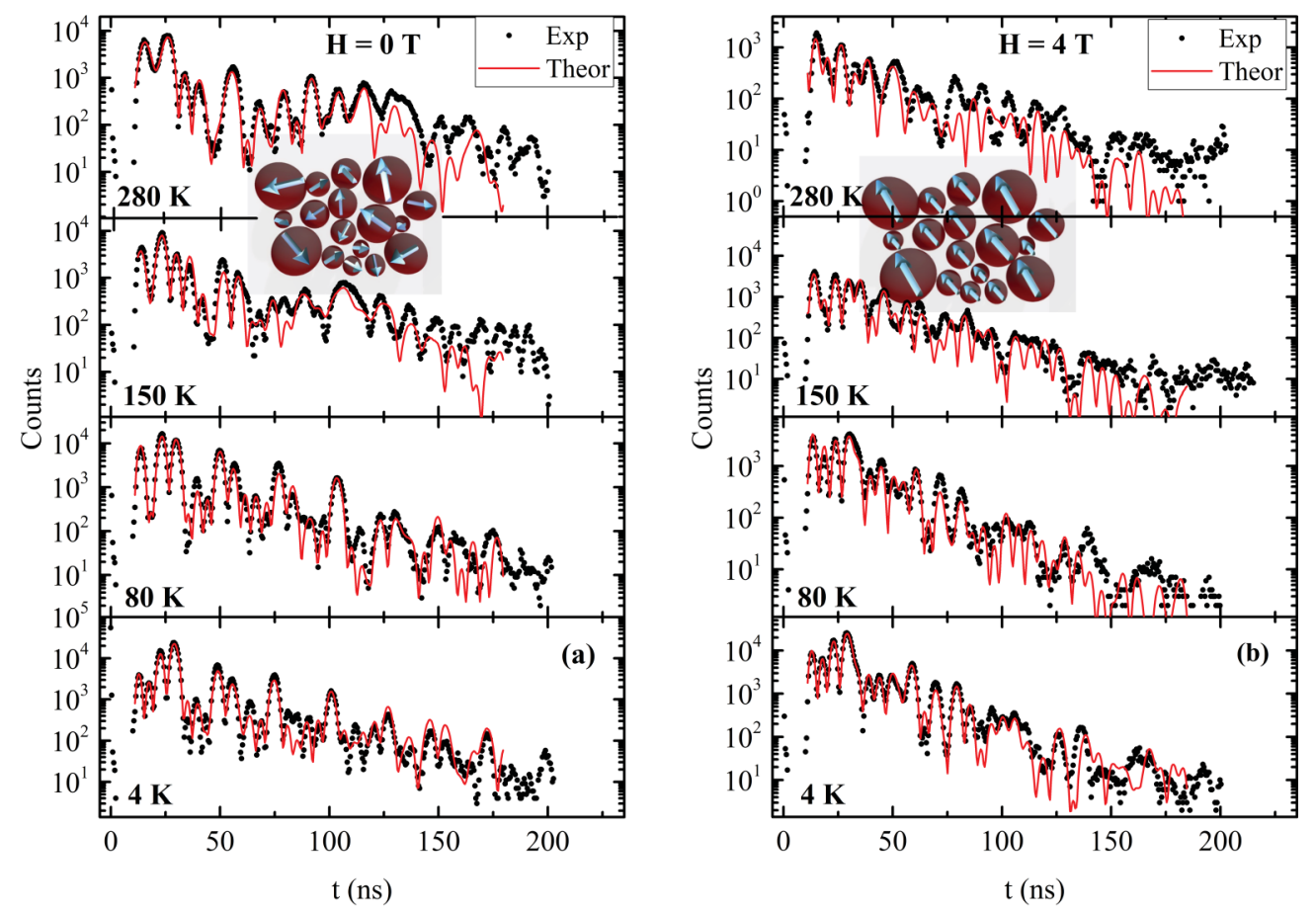

FIG. 2. Nuclear forward scattering spectra for the $\epsilon-\mathrm{Fe}_{2} \mathrm{O}_{3}$ nanoparticles with the average size of $8 \mathrm{~nm}$ in the temperature range of 4-300 $\mathrm{K}$ (a) without external magnetic field and (b) in a magnetic field of $4 \mathrm{~T}$. The inserts show the schematic configuration of the magnetic moments of nanoparticles.

reveal quantum beats resulting from the interference of electromagnetic waves emitted by different hyperfine components of the nuclear transition with similar resonance frequencies [22]. The shape of the spectra is determined by both the magnetic state of iron nuclei and the magnetic structure of the samples.

The spectra were processed by fitting the quantum beats with a number of decaying in time harmonic wave components using the MOTIF package [26], taking into account the experimental geometry, polarization of synchrotron radiation, and the direction of the external magnetic field. The MOTIF package simulates the NFS spectra by direct calculations of the first-order integrodifferential wave equation in space and time. The solution of the wave equation was obtained using a generalized procedure independent of the explicit form of hyperfine nuclear interactions. The hyperfine parameters of iron nuclei corresponding to those in the conventional Mössbauer spectroscopy were obtained for each nonequivalent site of the sample using the Fourier transformation of the time spectra. In addition, the procedure makes it possible to determine the angle between the vectors of the nuclear hyperfine field and the magnetic field applied to the sample in the geometry shown in Fig. 1. Since the applied magnetic field is stronger than the magnetic anisotropy of the sample, we assume that the particle size distribution does not make a significant contribution to the analysis of the NFS spectra.

\section{RESULTS}

The NFS spectra measured on nanoparticles with an average size of $8 \mathrm{~nm}$ under different temperature and magneticfield conditions are presented in Fig. 2. At $280 \mathrm{~K}$ in zero magnetic field, the spectrum shows the presence of nanoparticles in the superparamagnetic state. This is reflected as a coherent superposition of two types of the quantum beats, specifically, the fast quantum beats for particles with a hyperfine magnetic structure and the slow quantum beats of the quadrupole transitions in superparamagnetic particles. The coexistence of the two states in this system was observed also by conventional Mössbauer spectroscopy [27]. Upon cooling down, the contribution of the slow beats decreases due to the blocking of the magnetic moments of superparamagnetic particles and resolving the hyperfine magnetic structure. At $4 \mathrm{~K}$ without magnetic field, all nanoparticles pass to the blocked state. The spectra are typical of the isotropic-in-space magnetization, which is consistent with the random spatial orientation of the magnetic moments of individual noninteracting nanoparticles [28].

The absence of magnetic interactions between the $\epsilon-\mathrm{Fe}_{2} \mathrm{O}_{3}$ nanoparticles can be explained by their isolation in the xerogel matrix due to features of the synthesis of the material [23]. Without interactions, the directions of magnetic moments of the particles are only determined by their magnetic anisotropies and the random orientation of the particles in space leads to the isotropic magnetization of the sample. Then, any transformation of the magnetic structure during the magnetic transition cannot be observed by NFS. However, the problem can be overcome by applying a magnetic field, which effectively aligns the magnetic moments of particles along its direction [Fig. 2(b)]. As was shown in this study, the required alignment of the magnetic moments of singledomain particles is obtained already in an applied field of $2 \mathrm{~T}$. Since no features in the behavior of magnetization as a function of magnetic field are observed up to $7 \mathrm{~T}$ [23], we do 


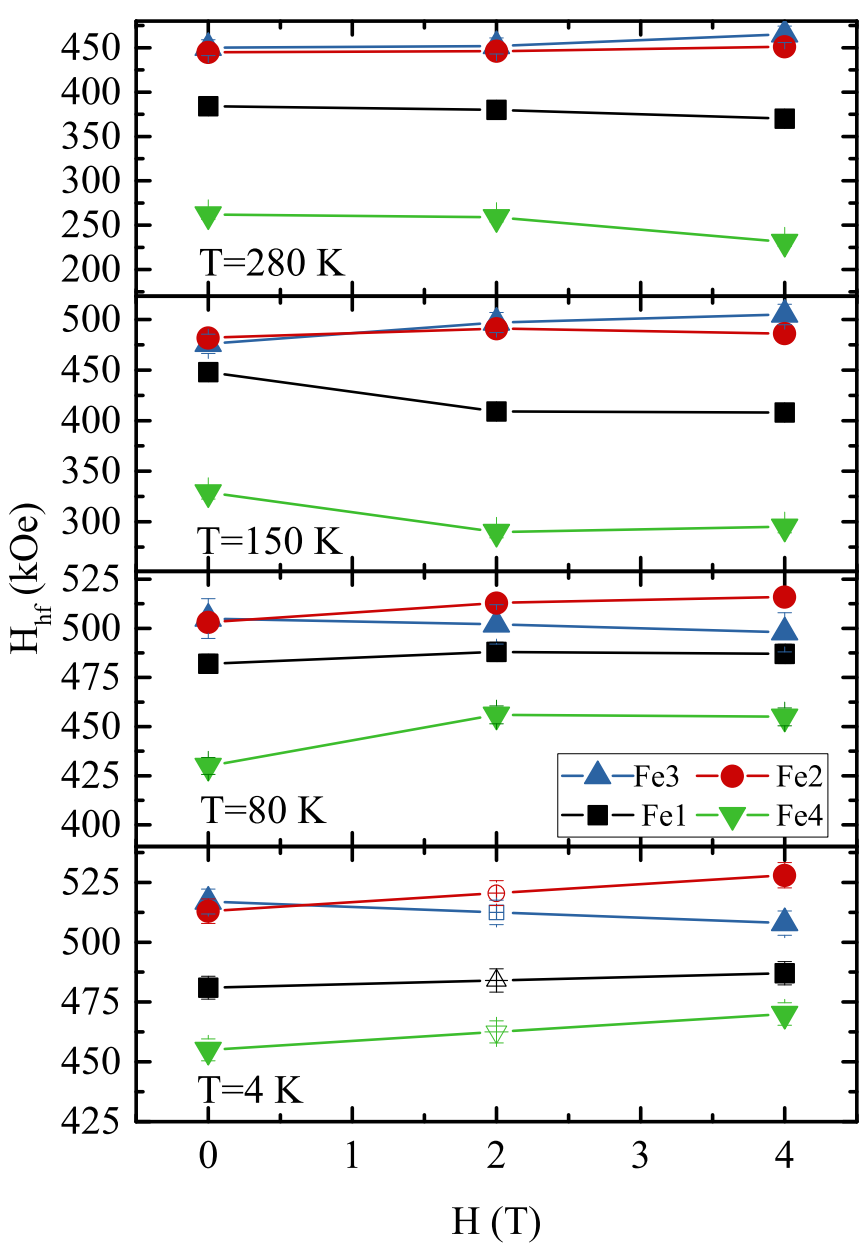

FIG. 3. Hyperfine field in four nonequivalent iron sites as a function of the external magnetic field at different temperatures in the range of 4-280 K. At $4 \mathrm{~K}$, open symbols show the interpolation of the experimental data by a linear function. Error bars are shown.

not expect any significant change in the magnetic structure of the sample in our experiment.

The NFS spectra recorded in a magnetic field of $4 \mathrm{~T}$ reveal significant changes with decreasing temperature [Fig. 2(b)]. The data processing (see below) shows that this is not related to the blocking of the magnetic moments of an ensemble of nanoparticles, but follows from the transformation of the $\epsilon-\mathrm{Fe}_{2} \mathrm{O}_{3}$ magnetic structure. The changes are especially pronounced in the time interval of 20-40 ns. Below the temperature of the magnetic transition, the additional quantum beats occurring in this time window indicate the transformation of the magnetic structure of the sample.

The hyperfine parameters of the nanoparticles obtained from the time spectra using the MOTIF package are consistent with the values determined previously by conventional Mössbauer spectroscopy $[3,7,27]$, including those measured in an applied magnetic field $[4,29]$. Figure 3 shows the temperature evolution of the hyperfine field on iron nuclei upon variation in the external magnetic field. Our results are somewhat different from the data reported in Ref. [29], where the effect of the magnetic transition in an applied magnetic field was only observed as a change in the $H_{\mathrm{hf}}(\mathrm{T})$ slope. In contrast

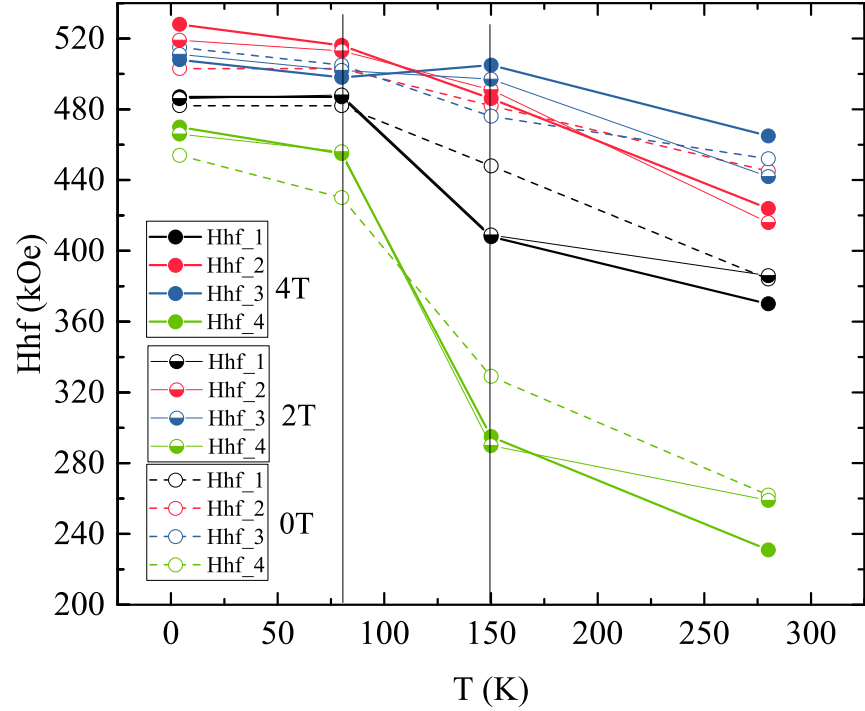

FIG. 4. Temperature dependence of the hyperfine field $H_{\mathrm{hf}}$ of the ultrafine $\epsilon-\mathrm{Fe}_{2} \mathrm{O}_{3}$ nanoparticles in different external magnetic fields.

to this situation, our results reveal the obvious change in these dependences before and after the magnetic transition. In particular, in the temperature range of $280-150 \mathrm{~K}$, the hyperfine field in the $\mathrm{Fe} 1$ and $\mathrm{Fe} 4$ sites decreases with the applied field, whereas below $80 \mathrm{~K}$ it does the opposite (Fig. 3).

Figure 3 shows that the dependence of the nuclear hyperfine fields on the external magnetic field changes at the temperature of the magnetic transition. In the temperature range of $280-150 \mathrm{~K}$, the applied magnetic field decreases the hyperfine field in the $\mathrm{Fe} 1$ and $\mathrm{Fe} 4$ sites, but increases it in the two other sites, $\mathrm{Fe} 2$ and Fe3. This shows that, in the range of $280-150 \mathrm{~K}$, the magnetic moments in the $\mathrm{Fe} 1$ and $\mathrm{Fe} 4$ sites are antiparallel to those in the $\mathrm{Fe} 2$ and $\mathrm{Fe} 3$ sites, which is consistent with the results reported in Refs. [3,4]. However, after cooling to the temperature of the magnetic transition, i.e., below $150 \mathrm{~K}$, this trend qualitatively changes: The applied magnetic field decreases the nuclear hyperfine field in the $\mathrm{Fe} 3$ site, but increases it in all the other sites. This possibly witnesses the change in the orientation of the magnetic moments in the $\epsilon-\mathrm{Fe}_{2} \mathrm{O}_{3}$ structure.

The temperature dependences of the nuclear hyperfine fields in different applied magnetic fields are shown in Fig. 4. The sharp growth of the hyperfine field in the tetrahedral $\mathrm{Fe} 4$ site with decreasing temperature reported in Refs. [3,4,7] was also observed in our measurements. In addition, our data clearly demonstrate the effect of the external field. The external magnetic field does not qualitatively change the $H_{\mathrm{hf}}(\mathrm{T})$ behavior, but makes a strong opposite impact on the hyperfine field in the $\mathrm{Fe} 2$ and $\mathrm{Fe} 3$ sites, leading to the divergence of $H_{\text {hf }}(\mathrm{T})$ dependences below $80 \mathrm{~K}$. So far, this effect has not been reported in the literature.

Further insights into the magnetic structure can be made by analyzing the temperature dependence of the hyperfine field directions in different iron sites. The MOTIF processing of the NFS spectra shows that, in an applied magnetic field up to $4 \mathrm{~T}$, the hyperfine field directions are inclined to the external field axis at some polar angle $\Theta[$ Fig. 5(a)] and 

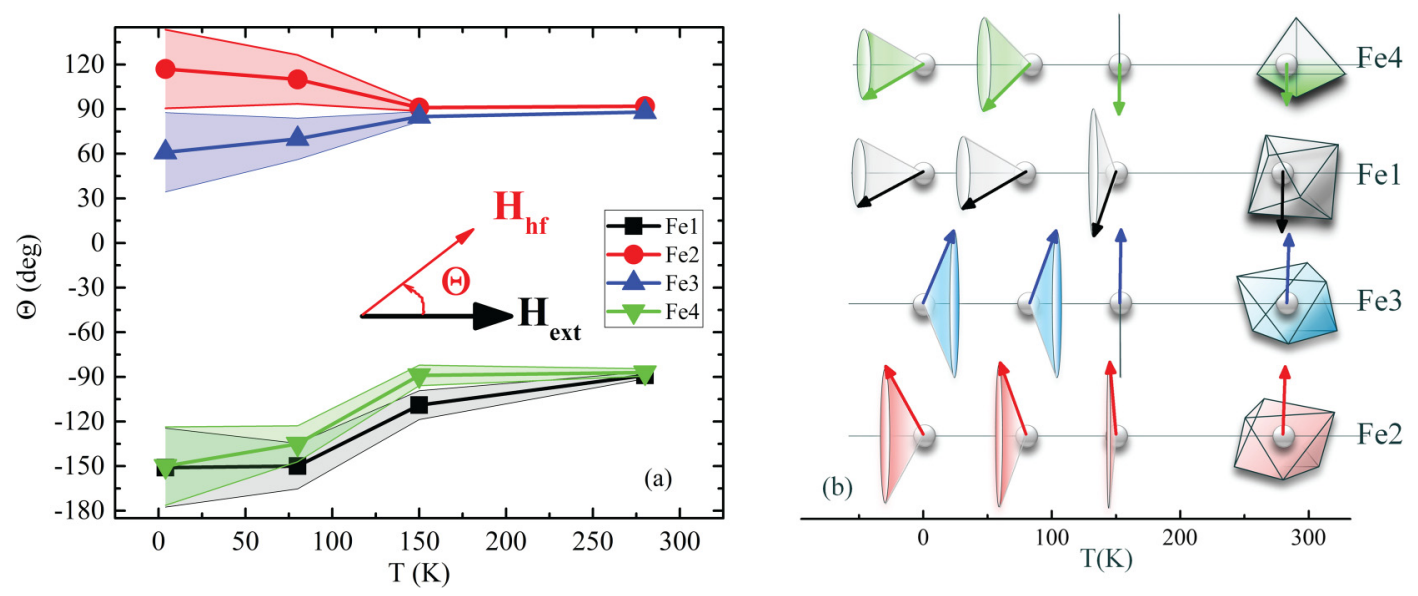

FIG. 5. Temperature dependence of the magnetic structure of $\epsilon-\mathrm{Fe}_{2} \mathrm{O}_{3}$ in an applied magnetic field of $\mathrm{H}=4 \mathrm{~T}$. (a) Polar angle $\Theta$ between the external magnetic field and the hyperfine field quantization axis in different iron sites. The solid lines show the average polar angles and the shaded areas show the divergence of the angles. The negative $\Theta$ values emphasize the orientations of the hyperfine fields in the Fe1 and $\mathrm{Fe} 4$ sites opposite to those in the $\mathrm{Fe} 2$ and Fe 3 sites (see the text). (b) Vector presentation of the magnetic structure.

randomly distributed over the azimuth angle around this axis. We note that the $\Theta$ values are almost independent of the applied magnetic field. Therefore, Fig. 5 shows the $\Theta$ values only for an external field of $4 \mathrm{~T}$. Thus, the magnetic moments of each sublattice form a cone with the opening given by the corresponding polar angle [Fig. 5(b)]. Within this random azimuthal distribution, however, the azimuthal angles of the hyperfine fields in the $\mathrm{Fe} 2$ and $\mathrm{Fe} 3$ sites are always opposite to those in the Fe1 and Fe4 sites [Fig. 5(b)]. In Fig. 5(a), this is emphasized by using positive values for the polar angles for the $\mathrm{Fe} 2$ and $\mathrm{Fe} 3$ sites and negative values for the Fe1 and $\mathrm{Fe} 4$ sites. The weak field dependence of the $\Theta$ value can be indicative of a minor impact of the applied field on the magnetic structure of the sample. In other words, the system satisfies the condition $E_{\mathrm{an}}<M H_{\mathrm{ext}}<E_{\mathrm{ex}}$, where $E_{\mathrm{an}}$ is the particle anisotropy energy, $M H_{\text {ext }}$ is the energy of the external magnetic field, and $E_{\mathrm{ex}}$ is the exchange energy of the sample. The angles determined in this study are consistent with the Mössbauer data obtained in an external magnetic field [3].

The analysis of the field dependences $H_{\mathrm{hf}}\left(H_{\mathrm{ext}}\right)$ (Fig. 3) and the NFS processing results allow us to distinguish the relative orientation (parallel or antiparallel) of the projection of the nuclear hyperfine field and external magnetic field vectors. In the temperature range of 4-80 K, the polar angles $\Theta$ for all iron sites are noticeably different from 90 deg [Fig. 5(a)]; i.e., the hyperfine magnetic fields are essentially inclined to the external field axis [Fig. 5(b)]. Under these conditions, a decrease in the hyperfine field in the $\mathrm{Fe} 3$ site with increasing external field is indicative of the antiparallel orientation of the $\mathbf{H}_{\text {hf }}$ projection and $H_{\text {ext }}$ vectors. For the $\mathrm{Fe} 1, \mathrm{Fe} 2$, and Fe4 sites, the field dependences (Fig. 3) show the opposite trend (increase with the field). Therefore, the vector of the $H_{\mathrm{hf}}$ projection for these sites is antiparallel to the applied field.

According to the results discussed above, the $\epsilon-\mathrm{Fe}_{2} \mathrm{O}_{3}$ magnetic structure in the temperature range of $4-300 \mathrm{~K}$ is summarized in Fig. 5. At $280 \mathrm{~K}, \epsilon-\mathrm{Fe}_{2} \mathrm{O}_{3}$ is a collinear ferrimagnet with the resulting magnetic moment determined by the uncompensated moments of the octahedral sites, in accordance with the results from Refs. [7,21]. A decrease in temperature leads to the canting of the magnetic moments and induces the noncollinear orientation of the hyperfine field projections below $150 \mathrm{~K}$. Since in the temperature interval of $150-80 \mathrm{~K}$ the orbital magnetic moment of iron in the Fe4 site shows a pronounced slippage [18], the energy of the spin-orbit coupling should behave similarly and the coupling between the magnetic moment and crystal field should weaken. This leads to the divergence of the $\mathrm{Fe} 4$ and $\mathrm{Fe} 1$ magnetic moments and it is what we can see from our NFS data (Fig. 5). Below the magnetic transition, in the temperature range of $80-4 \mathrm{~K}$, the orbital moment in the $\mathrm{Fe} 4$ site is almost equal to that observed above $150 \mathrm{~K}$. Thus, the spin-orbit coupling of the Fe4 iron is restored and the magnetic moment in this site is tightly coupled to the lattice field again. As a result, at $4 \mathrm{~K}$, the angles $\Theta$ in the $\mathrm{Fe} 1$ and $\mathrm{Fe} 4$ sites are almost equal $\left(148^{\circ}\right.$ and $150^{\circ}$, respectively).

In recent studies [30,31], it was shown by Mössbauer spectroscopy and XMCD in strong magnetic fields that the low-temperature magnetic structure is only established in the vicinity of $\approx 50 \mathrm{~K}$. Below $50 \mathrm{~K}$, the spin configuration of $\epsilon-\mathrm{Fe}_{2} \mathrm{O}_{3}$ becomes stable, which is consistent with our NFS data obtained in the temperature range of 80-4 K. Furthermore, the temperature dependence of magnetization contains a pronounced anomaly in the strong magnetic field (7 T) around $50 \mathrm{~K}$ (the arrow in Fig. 6). We suppose that the magnetic moment rotation in the $\mathrm{Fe} 4$ site in this temperature range can be reflected by this macroscopic $M(\mathrm{~T})$ feature.

The solution of the integrodifferential wave equation using the MOTIF package during the processing of the spectra obtained in an external magnetic field yields satisfactory results only when the analysis includes the spatial divergence of the magnetic moment in each iron site from the average axis. This parameter accounts for the angular divergence of the hyperfine field directions [shown by the shaded area in Fig. 5(a)] around the average direction (shown by the solid lines). Physically, this can be interpreted as the occurrence of an additional degree of freedom, which leads to a finite angular divergence for the magnetic moment vector. The observed increase in the angular divergence upon cooling [Fig. 5(a)] indicates 


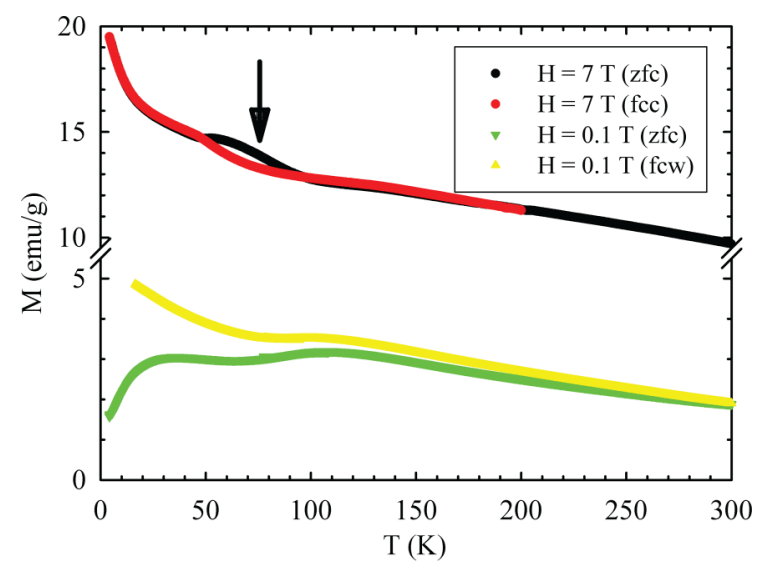

FIG. 6. Temperature dependences of magnetization $M(\mathrm{~T})$ of the sample in different magnetic fields for different thermomagnetic prehistories: zero field cooling (ZFC), field cooling (FCC), and field cooled warming (FCW). The magnetization is normalized to the iron oxide mass in the sample. The arrow shows the feature at $50 \mathrm{~K}$.

that, at lower temperatures, the noncollinear ordering of the magnetic moments transforms into the spiral structure, which agrees with the neutron diffraction data obtained at $10 \mathrm{~K}$ [7]. Our results show that, above $150 \mathrm{~K}$, the divergence of the magnetic moments remains zero for all the sites. At lower temperatures, the divergence of the directions of the magnetic vectors gradually opens around its average value and the temperature dependences of the opening are different for different nonequivalent sites [Fig. 5(a)]. Obviously, the formation of a spiral structure is completed only at $4 \mathrm{~K}$, where the divergence angle is identical for all the sites and equal to $55^{\circ}$.

Dealing with the polycrystalline samples, we cannot determine the wave vectors $\mathbf{k}$ of the spirals in the nonequivalent sites. However, the different temperatures of the formation of the spiral magnetic structure in different nonequivalent iron sites [Fig. 5(a)] could be indicative of different anisotropies in these sites. This is equivalent to the entire range of wave vectors k $\mathbf{k}$, i.e., to some modulated spiral structure, as was observed using neutron diffraction in Ref. [7]. Normally, the modulated structure is not a unique magnetically ordered phase of a magnetic material and there are allowed transitions between the modulated spiral structure and the commensurate magnetic phase. These transitions can be induced by varying the temperature or applying a magnetic field, as was observed, for example, in $\mathrm{CuB}_{2} \mathrm{O}_{4}$ [32]. Our results and the neutron diffraction data from Ref. [7] show the possibility of such a behavior in $\epsilon-\mathrm{Fe}_{2} \mathrm{O}_{3}$. The formation of such structures and magnetic transitions between them are often caused by frustration of the magnetic structure (see below).

\section{DISCUSSION}

The drastic growth of the hyperfine field in the tetrahedral $\mathrm{Fe} 4$ sites upon cooling the sample in the temperature range of $80-150 \mathrm{~K}$ was observed in our (Fig. 4) and other $\epsilon-\mathrm{Fe}_{2} \mathrm{O}_{3}$ samples $[4,7,18]$. On the one hand, the significant distortions of the $\epsilon-\mathrm{Fe}_{2} \mathrm{O}_{3}$ crystal lattice result in the elongation of the

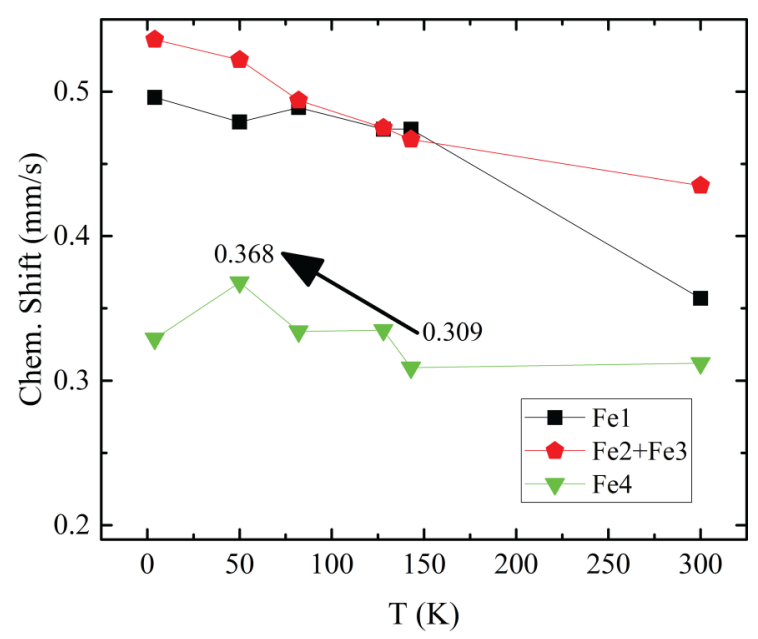

FIG. 7. Temperature dependence of the chemical shift in $\epsilon-\mathrm{Fe}_{2} \mathrm{O}_{3}$ measured by conventional Mössbauer spectroscopy. Adapted from Ref. [19].

Fe-O bonds for the Fe4 sites by $2 \%$ [7]. On the other hand, the iron orbital moment decreases in this temperature range [18]. The elongation of the bond reduces the probability of admixing an oxygen electron. Therefore, we may assume that the orbital moment of the ferric iron is induced by admixing of $2 p$ oxygen electrons to the iron $e_{g}$ orbitals, which have the lower energy in the tetrahedral surrounding. The sharp growth of the local distortions in the tetrahedral sites leads to the divergence of the iron $e_{g}$ orbitals in energy. Therefore, the probability of electron hopping to these orbitals decreases and the induced orbital moment of iron in the tetrahedral sites decreases as well. On the contrary, during this process, the effective magnetic moment increases, which leads to an increase in the hyperfine field due to the enhancement of the polarization of electrons in the iron $s$ shells. This effect was also observed when studying the temperature dependence of the orbital moment in Refs. [7,18]. This explanation is confirmed by the increasing chemical shift for the Fe4 site upon cooling below $150 \mathrm{~K}$ (shown by the arrow in Fig. 7), which is caused by a decrease in the electron density on iron nuclei, as was observed both in this experiment and using conventional Mössbauer spectroscopy [19,27].

In studies [33,34], it was shown that the formation of the noncollinear and incommensurate magnetic structures in ferrimagnets is accompanied by the magnetoelectric effect. In our case, the abrupt change in the orbital moment and formation of the noncollinear magnetic structure occur in the same temperature range. This correlation leads to the magnetoelectric coupling in $\epsilon-\mathrm{Fe}_{2} \mathrm{O}_{3}$, which was reported in Ref. [20]. Such a magnetoelectric coupling was not observed in other nonsubstituted ferrimagnetic metal oxides. Study of the origin of the noncollinear magnetic structure in $\epsilon-\mathrm{Fe}_{2} \mathrm{O}_{3}$ may bring the light into the mechanism of the magnetoelectric effect.

The possible reasons for the formation of noncollinear and spiral magnetic structures in $\epsilon-\mathrm{Fe}_{2} \mathrm{O}_{3}$ were discussed in Ref. [21], where a model of the noncollinear magnetic structure at $300 \mathrm{~K}$ based on the calculated indirect exchange interactions between the $\epsilon-\mathrm{Fe}_{2} \mathrm{O}_{3}$ sublattices was proposed. However, the interactions within the magnetic sublattices 


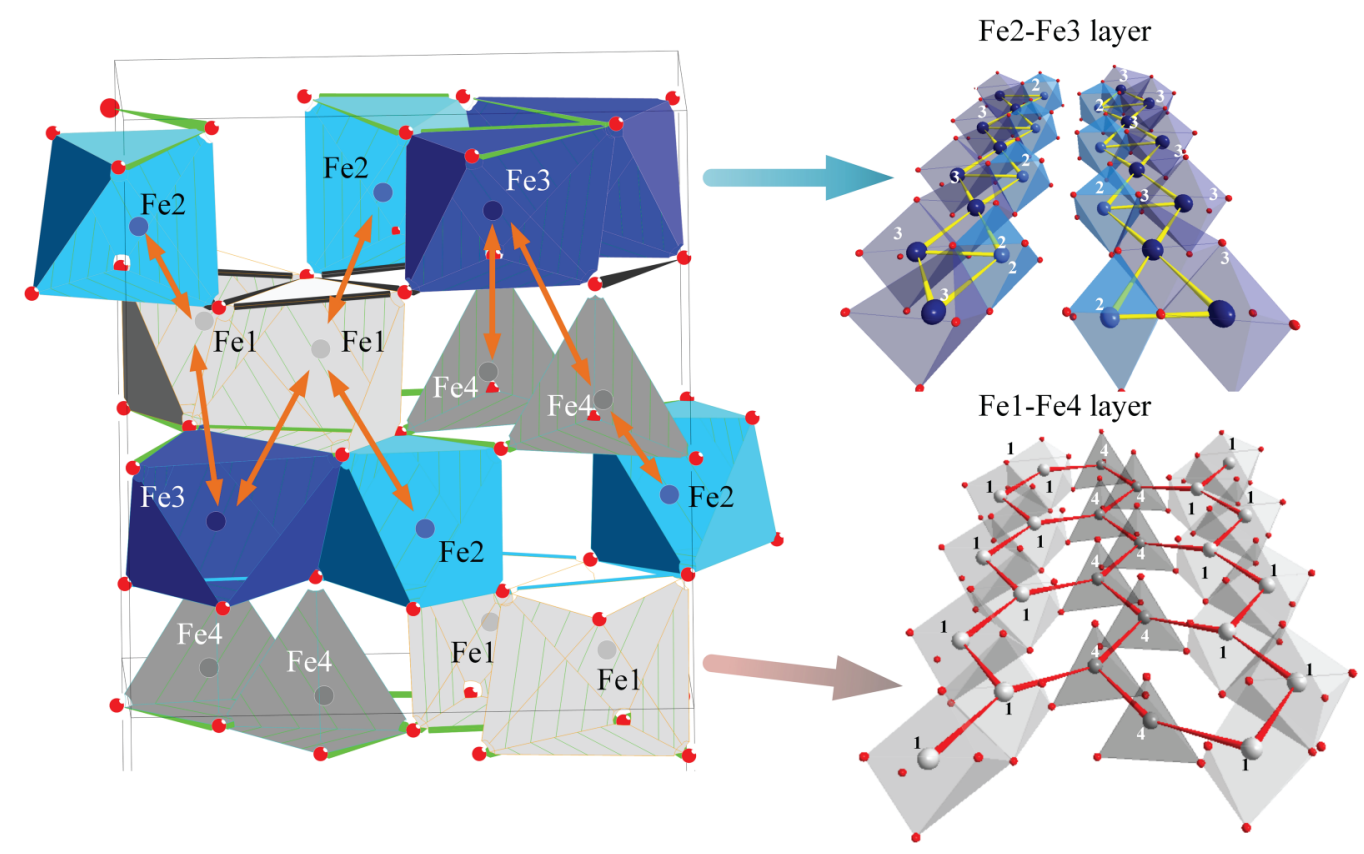

FIG. 8. Layered $\epsilon-\mathrm{Fe}_{2} \mathrm{O}_{3}$ structure with the indicated crystallographic sites of the iron atoms. The bold lines show the indirect exchange interactions within the layers. The interactions between the layers are shown by arrows.

were not considered. Therefore, the proposed model might not reflect the effect of the frustration in the $\epsilon-\mathrm{Fe}_{2} \mathrm{O}_{3}$ magnetic structure. Generally, the frustration is one of the causes of the formation of incommensurate long-period modulated spiral structures [35], which was observed by us in $\epsilon-\mathrm{Fe}_{2} \mathrm{O}_{3}$ by the NFS measurements. Here, we evaluated the indirect exchanges taking into account also the interactions within the sublattices using the indirect coupling model from Refs. [36-38].

The crystal structure of $\epsilon-\mathrm{Fe}_{2} \mathrm{O}_{3}$ is well studied [1,2,7]. It consists of the layers of two types formed by different iron crystallographic sites (Fig. 8). The type-1 layers consist of the triple chains of $\mathrm{Fe} 1$ octahedra and single chains of Fe4 tetrahedra. The type-2 layers include the chains of $\mathrm{Fe} 2$ and $\mathrm{Fe} 3$ octahedra. These chains are shown in Fig. 8. The bold lines show the indirect exchange interactions forming the magnetic structure of $\epsilon-\mathrm{Fe}_{2} \mathrm{O}_{3}$. The chains have some features, which can affect the magnetic properties of the material and manifest themselves during the magnetic transitions. The cations in the tetrahedral $\mathrm{Fe} 4$ sites together with the octahedral Fe1 sites form a honeycomb-like structure of the indirect exchange interactions.

The most striking feature of the $\mathrm{Fe} 2-\mathrm{Fe} 3$ layers is the absence of magnetic interactions between cations in the Fe2 sites. This leads to the separation of the chains of octahedra in two uncoupled chains (Fig. 8). These chains form exchange couplings with the triangular arrangement of cations, which inevitably leads to magnetic frustrations in the structure. Therefore, the magnetic cations in these chains can have a noncollinear orientation of the magnetic moments.

Despite the seeming complexity, the entire set of the exchange interactions in this system can be characterized by four types of interactions, which are described by the corresponding integrals. The interactions of cations in the edge-sharing octahedral sites are of $90^{\circ}$ character. They can be expressed as

$$
J_{O-O}=\frac{1}{4 S_{1} S_{2}}\left[-2 U\left(\frac{16}{3} b c+2 c^{2}\right)\right] .
$$

The interaction between cations through the common vertices of octahedra is described as

$$
J_{O-O}^{180^{\circ}}=\frac{1}{4 S_{1} S_{2}}\left[-2 U\left(\frac{16}{9} b^{2}+2 c^{2}\right)\right]|\cos \theta| .
$$

The interactions between the tetrahedral and octahedral sites are expressed in the form

$$
J_{O-T}=\frac{1}{4 S_{1} S_{2}}\left[-2 U\left(\frac{4}{3} a b+2 a c\right)\right]|\cos \theta| .
$$

The indirect exchange interaction between $\mathrm{Fe} 3+$ cations in the tetrahedral positions is described by the integral

$$
J_{T-T}=\frac{1}{4 S_{1} S_{2}}\left[-3 a^{2} U\right] \cos \theta^{2} .
$$

Here, $\theta$ is the angle of the exchange interactions, which can easily be determined from the x-ray diffraction data [1,7]; $S_{1}$ and $S_{2}$ are the spins of the interacting cations; $U=8 \mathrm{eV}$ is the of energy of the electron excitation between oxygen and the $\mathrm{Fe}^{3+}$ cation; $a \approx 0.12$ is the parameter of the electron transport (covalence) for iron in the tetrahedral coordination; and $b \approx 0.024$ and $c \approx 0.012$ are the analogous parameters for the octahedral surrounding of iron along the $\sigma$ and $\pi$ bonds, respectively. These values are similar to the corresponding covalence parameters for spinel ferrites [38], where the average metal-oxygen distances are similar to those in $\epsilon-\mathrm{Fe}_{2} \mathrm{O}_{3}$ [20].

The exchange interactions calculated using to the above equations with regard to the number of nearest magnetic neighbors and $\mathrm{Fe}-\mathrm{O}-\mathrm{Fe}$ bond angles are given in Table I. The account for the interaction within the magnetic sublattices 
TABLE I. Calculated values of the indirect exchange interactions in $\epsilon-\mathrm{Fe}_{2} \mathrm{O}_{3}(\mathrm{~K})$. The frustrating interactions are highlighted in bold. Arrows show the relative magnetic moments' directions.

\begin{tabular}{ccccc}
\hline \hline & $1 \downarrow$ & $2 \uparrow$ & $3 \uparrow$ & $4 \downarrow$ \\
\hline $1 \downarrow$ & $\mathbf{2 7}$ & -40 & -43 & $\mathbf{- 1 8}$ \\
$2 \uparrow$ & -40 & 0 & $\mathbf{- 4 1}$ & -102 \\
$3 \downarrow$ & -43 & -41 & $\mathbf{- 2 7}$ & -75 \\
$4 \downarrow$ & $\mathbf{- 1 8}$ & -102 & -75 & $\mathbf{- 8 2}$ \\
\hline \hline
\end{tabular}

makes it clear that the antiferromagnetic character of these interactions and the similarity of their values to those of the intersublattice interactions in the order of magnitude (except for the noninteracting $\mathrm{Fe} 2$ cations) play an important role in the formation of the magnetic structure. The triangular arrangement of the cations [39] leads to a high level of frustrations in the system. Our estimation of the level of frustrations in the investigated magnetic system yields a relative value of $40-55 \%$ for the $\mathrm{Fe} 3$ and $\mathrm{Fe} 4$ sites. A huge contribution of the frustrations to the magnetism in $\epsilon-\mathrm{Fe}_{2} \mathrm{O}_{3}$ was established using the density functional theory in Ref. [40]. Yafet and Kittel showed [41,42] that there is a boundary condition for the existence of the collinear configuration of the magnetic moments. It is determined by the parameter $u=\frac{4 J_{j j} S_{j}}{3 J_{i j} S_{i}}$, where $i$ and $j$ are the indices of the magnetic sublattices, $J_{i j}$ is the integral of the exchange interaction between the sublattices, and $S_{i}$ and $S_{j}$ are the spins of the sublattices, which, in our case, are equal to each other. According to Kaplan's criterion [35], the collinear configuration for tetragonal spinels exists below the critical value of this parameter $u=\frac{8}{9}$. For $\epsilon-\mathrm{Fe}_{2} \mathrm{O}_{3}$, the value of this parameter for the magnetic sublattice of a tetrahedron is much higher: $u=1.46$. This suggests not only the canting of the magnetic moments, but also the possible formation of a spiral magnetic structure as a ground state of the $\epsilon-\mathrm{Fe}_{2} \mathrm{O}_{3}$ system. Upon heating, such a structure should collapse and transform to a more symmetric collinear magnetic structure. This scenario confirms the temperature evolution of the magnetic structure suggested by our NFS data and the results of the neutron diffraction study [7]. This conclusion is indirectly confirmed by the existence of a paraprocess in the temperature dependence of magnetization and its nonmonotonic temperature evolution (Fig. 6). The possible transitions from the Yafet-Kittel's canted structure to the noncollinear spiral magnetic structures predicted already in Ref. [39] were considered in theoretical studies [43,44]. The results obtained showed that the noncollinear configuration of the magnetic moments can transform to the state incommensurate with the crystal lattice cell at a certain frustration level $[43,44]$. This was possibly observed in the neutron diffraction experiments [7], which revealed the formation of such a state. Thus, one can distinguish two main factors contributing to the occurrence of the incommensurate magnetic structure in $\epsilon-\mathrm{Fe}_{2} \mathrm{O}_{3}$. First, iron atoms in the $\mathrm{Fe} 2$ and $\mathrm{Fe} 3$ sites form noncoupled ribbons with the triangular arrangement of cations, which leads to the strong frustrations of the magnetic system. Second, the strong antiferromagnetic exchange interactions between the $\mathrm{Fe} 4$ and $\mathrm{Fe} 3$ sites result from the occurrence of the Yafet-Kittel magnetic configurations between layers, which can transform into the incommensurate spiral magnetic structure with a decrease in temperature.

\section{CONCLUSIONS}

In summary, we used a synchrotron radiation source to investigate the magnetic structure of the nanoscale $\epsilon-\mathrm{Fe}_{2} \mathrm{O}_{3}$ compound. We obtained time spectra of nuclear forward scattering in the temperature range of $4-300 \mathrm{~K}$ for isolated nanoparticles with an average size of $8 \mathrm{~nm}$ immobilized in a xerogel matrix. Furthermore, we applied a magnetic field of $0-4 \mathrm{~T}$ in the longitudinal direction for the first time. The results of the polarized synchrotron radiation investigations of the isolated $\epsilon-\mathrm{Fe}_{2} \mathrm{O}_{3}$ nanoparticles in a xerogel matrix show that the magnetic structure of the system is collinear at room temperature. However, as a result of the strong frustrations of the magnetic structure, a cascade of magnetic transitions occurs upon cooling, which leads to the formation of the noncollinear magnetic structure with the magnetic spiral as the ground state of the system. The experimental results were confirmed by the estimation of the indirect exchange interactions and the existence of a paraprocess in the temperature dependence of magnetization, where the susceptibility changes nonmonotonically.

\section{ACKNOWLEDGMENTS}

We thank Dr. Natalia Kazak for assistance with the NFS measurements. This study was supported by the Russian Science Foundation, Project No. 17-12-01111. We thank the European Synchrotron Radiation Facility for provision of the synchrotron radiation facilities at the beamline ID18 (Exp. No. SC-4708).
[1] E. Tronc, C. Chanéac, and J. Jolivet, J. Solid State Chem. 139, 93 (1998)

[2] K. Kelm and W. Mader, Z. Anorg. Allg. Chem. 631, 2383 (2005).

[3] M. Kurmoo, J.-L. Rehspringer, A. Hutlova, C. D’Orléans, S. Vilminot, C. Estournès, and D. Niznansky, Chem. Mater. 17, 1106 (2005).

[4] J.-L. Rehspringer, S. Vilminot, D. Niznansky, K. Zaveta, C. Estournès, and M. Kurmoo, in ICAME 2005 (Springer, Berlin, 2006), pp. 475-481.
[5] M. Popovici, M. Gich, D. Nižňanský, A. Roig, C. Savii, L. Casas, E. Molins, K. Zaveta, C. Enache, J. Sort et al., Chem. Mater. 16, 5542 (2004).

[6] V. N. Nikolić, M. Tadić, M. Panjan, L. Kopanja, N. Cvjetićanin, and V. Spasojević, Ceramics Int. 43, 3147 (2017).

[7] M. Gich, C. Frontera, A. Roig, E. Taboada, E. Molins, H. Rechenberg, J. Ardisson, W. Macedo, C. Ritter, V. Hardy et al., Chem. Mater. 18, 3889 (2006).

[8] V. N. Nikolic, M. M. Milic, J. D. Zdravkovic, and V. Spasojevic, Russ. J. Phys. Chem. A 93, 588 (2019). 
[9] J.-L. Garcia-Muñoz, A. Romaguera, F. Fauth, J. Nogués, and M. Gich, Chem. Mater. 29, 9705 (2017).

[10] D. Balaev, I. Poperechny, A. Krasikov, K. Shaikhutdinov, A. Dubrovskiy, S. Popkov, A. Balaev, S. Yakushkin, G. Bukhtiyarova, O. Martyanov et al., J. Appl. Phys. 117, 063908 (2015).

[11] M. Gich, A. Roig, C. Frontera, E. Molins, J. Sort, M. Popovici, G. Chouteau, D. Martín y Marero, and J. Nogues, J. Appl. Phys. 98, 044307 (2005).

[12] S. Yakushkin, A. Dubrovskiy, D. Balaev, K. Shaykhutdinov, G. Bukhtiyarova, and O. Martyanov, J. Appl. Phys. 111, 044312 (2012).

[13] A. Dubrovskiy, D. Balaev, A. Krasikov, S. Yakushhkin, V. Kirillov, and O. Martyanov, Solid State Commun. 289, 27 (2019).

[14] J. Tuček, P. Tuček, J. Čuda, J. Filip, J. Pechoušek, L. Machala, and R. Zbořil, in AIP Conf. Proc. (AIP, New York, 2012), Vol. 1489, pp. 56-74.

[15] R. Zboril, M. Mashlan, and D. Petridis, Chem. Mater. 14, 969 (2002).

[16] S. Sakurai, J.-i. Shimoyama, K. Hashimoto, and S.-I. Ohkoshi, Chem. Phys. Lett. 458, 333 (2008).

[17] M. Gich, J. Gazquez, A. Roig, A. Crespi, J. Fontcuberta, J. Idrobo, S. Pennycook, M. Varela, V. Skumryev, and M. Varela, Appl. Phys. Lett. 96, 112508 (2010).

[18] Y.-C. Tseng, N. M. Souza-Neto, D. Haskel, M. Gich, C. Frontera, A. Roig, M. Van Veenendaal, and J. Nogués, Phys. Rev. B 79, 094404 (2009).

[19] Y. V. Knyazev, D. Balaev, V. Kirillov, O. Bayukov, and O. Mart'yanov, JETP Lett. 109, 613 (2019).

[20] J. Tucek, R. Zboril, A. Namai, and S.-I. Ohkoshi, Chem. Mater. 22, 6483 (2010).

[21] S.-I. Ohkoshi, A. Namai, and S. Sakurai, J. Phys. Chem. C 113, 11235 (2009).

[22] G. Smirnov, Hyperfine Interact. 125, 91 (2000).

[23] S. Yakushkin, D. Balaev, A. Dubrovskiy, S. Semenov, Y. V. Knyazev, O. Bayukov, V. Kirillov, R. Ivantsov,
I. Edelman, and O. Martyanov, Ceramics Int. 44, 17852 (2018).

[24] R. Rüffer and A. I. Chumakov, Hyperfine Interact. 97, 589 (1996).

[25] A. Q. Baron, Hyperfine Interact. 125, 29 (2000).

[26] Y. V. Shvyd'ko, Hyperfine Interact. 125, 173 (2000).

[27] Y. V. Knyazev, D. Balaev, V. Kirillov, O. Bayukov, and O. Mart'yanov, JETP Lett. 108, 527 (2018).

[28] R. Röhlsberger, J. Bansmann, V. Senz, K. L. Jonas, A. Bettac, K. H. Meiwes-Broer, and O. Leupold, Phys. Rev. B 67, 245412 (2003).

[29] J. Kohout, P. Brázda, K. Závěta, D. Kubániová, T. Kmječ, L. Kubíčková, M. Klementová, E. Šantavá, and A. Lančok, J. Appl. Phys. 117, 17D505 (2015).

[30] R. Jones, R. Nickel, P. K. Manna, J. Hilman, and J. van Lierop, Phys. Rev. B 100, 094425 (2019).

[31] A. A. Dubrovskiy, S. V. Semenov, Y. V. Knyazev, S. I. Popkov, S. S. Yakushkin, V. L. Kirillov, O. N. Martyanov, and D. A. Balaev, IEEE Mag. Lett. 10, 1 (2019).

[32] M. Boehm, B. Roessli, J. Schefer, B. Ouladdiaf, A. Amato, C. Baines, U. Staub, and G. Petrakovskii, Phys. B (Amsterdam, Neth.) 318, 277 (2002).

[33] T. Kimura, Annu. Rev. Mater. Res. 37, 387 (2007).

[34] Y. Tokura and S. Seki, Adv. Mater. 22, 1554 (2010).

[35] T. Kaplan, Phys. Rev. 116, 888 (1959).

[36] P. W. Anderson, in Solid State Physics, Vol. 14 (Elsevier, Amsterdam, 1963), pp. 99-214.

[37] M. V. Eremin, Sov. Phys. Solid State 24, 239 (1982).

[38] O. Bayukov and A. Savitskii, Phys. Status Solidi B 155, 249 (1989).

[39] R. Moessner and A. P. Ramirez, Phys. Today 59(2), 24 (2006).

[40] K. Xu, J. S. Feng, Z. P. Liu, and H. J. Xiang, Phys. Rev. Appl. 9, 044011 (2018).

[41] Y. Yafet and C. Kittel, Phys. Rev. 87, 290 (1952).

[42] J. B. Goodenough, Magnetism and Chemical Bond, Vol. 1 (Interscience, New York, 1963).

[43] S. N. Martynov, JETP Lett. 95, 188 (2012).

[44] S. N. Martynov, JETP Lett. 102, 100 (2015). 